\title{
COMPARISON OF LIPID PROFILE IN PATIENTS WITH DIABETIC NEUROPATHY AND WITHOUT NEUROPATHY; A CROSS SECTIONAL STUDY
}

\author{
Kanwal Huma, Khadija Mehboob, Amir Rashid, Fatima Fuad*, Muhammad Mamoon Mujahid**, Asma Arshad***
}

Army Medical College/National University of Medical Sciences (NUMS) Rawalpindi Pakistan, *Islamabad Medical and Dental College, Islamabad Pakistan, **Pak Emirates Military Hospital/National University of Medical Sciences (NUMS) Rawalpindi Pakistan, ${ }^{* * * C o m b i n e d ~}$ Military Hospital Lahore/National University of Medical Sciences (NUMS) Pakistan

\begin{abstract}
Objective: To compare lipid profile in patients with diabetic neuropathy and without neuropathy. Study Design: Cross-sectional comparative study.

Place and Duration of Study: This study was carried out at Multidisciplinary Lab-1, Department of Biochemistry and Molecular Biology, Army Medical College, from Jan 2020 to Sep 2020 in collaboration with Pak-Emirates Military Hospital, Rawalpindi.

Methodology: Eighty-four subjects were selected and divided into three groups, twenty-eight in each. Group I included diagnosed patients with type 2 diabetes mellitus with peripheral neuropathy, group II included diagnosed patients with type 2 diabetes mellitus without peripheral neuropathy and group III consisted of healthy individuals. Biochemical parameters including total cholesterol, triglycerides, high density lipoprotein cholesterol and low density lipoprotein cholesterol were measured. Data was analyzed by SPSS version 22.0. One way ANOVA test was used that followed by post-Hoc Tukey test for group comparison.

Results: There was significant difference in triglycerides ( $p$-value 0.001) and high density lipoprotein cholesterol (0.003) levels among group I and III. While, between group II and III significant difference of high density lipoprotein cholesterol was present. There was no significant difference between group I and II regarding all lipid profile parameters.

Conclusion: There was significant increase of triglycerides levels and decrease of high density lipoprotein cholesterol in patients with diabetic peripheral neuropathy as compared to healthy controls. No significant difference was observed for all parameters of lipid profile between patients with diabetic peripheral neuropathy and without peripheral neuropathy.
\end{abstract}

Keywords: Diabetic peripheral neuropathy, Lipid profile, Type 2 diabetes mellitus.

This is an Open Access article distributed under the terms of the Creative Commons Attribution License (http://creativecommons.org/licenses/by/4.0), which permits unrestricted use, distribution, and reproduction in any medium, provided the original work is properly cited.

\section{INTRODUCTION}

Diabetes mellitus (DM) is a complex, chronic metabolic disorder recognized as of the top ten causes of deaths in adults. Around 463 million individuals are currently living with DM worldwide. This number will further increase up to 578 million in 2030. The prevalence of DM in Pakistan is $19.4 \%$ and the country is ranked fourth among top ten countries with DM. Moreover, Pakistan is expected to surpass the United States of America, and move to third place by $2045^{1}$. Long-standing type 2 diabetes mellitus with poor glycemic control eventually causes macrovascular and microvascular complications such as neuropathy, nep-

Correspondence: Dr Kanwal Huma, Biochemistry Department, Army Medical College Rawalpindi Pakistan

Received: 14 Oct 2020; revised received: 28 Oct 2020; accepted: 10 Nov 2020 hropathy and retinopathy².

Diabetic neuropathies are most common microvascular complications as they affect approximately $30-90 \%$ of DM patients in the world ${ }^{3}$. According to American diabetes association, there are three main types of diabetic neuropathy. These are diffuse neuropathy, mononeuropathy (mononeuritis multiplex) and radiculopathy or polyradiculopathy. Among diffuse neuropathies, distal symmetrical peripheral neuropathy (DSPN) is most common variety and generally referred to as diabetic peripheral neuropathy (DPN) or diabetic neuropathy (DN). So, the present study is focused on diabetic peripheral neuropathy. DPN is present in $10-15 \%$ of patients with type II diabetes mellitusat time of their diagnosis and this rate is further increased up to $50 \%$ 
after 10 years of their diagnosis of $\mathrm{DM}^{4}$. It is defined as symmetrical, length-dependent sensorymotor polyneuropathy that is caused by microvasculature and metabolic alterations as a result of chronic hyperglycemia ${ }^{5}$.

Hyperglycemia, insulin resistance and dyslipidemia are main pathological factors that involved in nerve dysfunction and cellular death in diabetic neuropathy. They activate multiple biochemical pathways such as polyol, hexosamine pathways and loss of insulin signaling, which cause oxidative stress, inflammation and mitochondrial dysfunction and alter gene expression. These altered metabolic changes not only affect nerves but also disturb mitochondrial redox balance and increased production of mitochondrial and cytosolic reactive oxygen species ${ }^{6}$. While, hyperlipidemia causes production of pro-inflammatory substances from adipocytes ${ }^{7}$.

Many studies reported the dyslipidemia in patients with type $2 \mathrm{DM}$ is characterized by elevated levels of triacylglycerol and reduced levels of high-density lipoprotein (HDL) cholesterol and these alterations may be reported several years before the clinical detection of hyperglycemia ${ }^{8}$. Increased triacylglycerol levels are associated with development of neuropathy in type 2 diabetes while obesity is independently correlated with development of neuropathy. Some studies report that lipid lowering drugs reduce the risk of lower-limb amputations, which is one of the most serious and life altering complications of diabetic neuropathy .

Deranged lipid profile in patients with diabetes mellitus result either of diabetic complication or as a risk factor for diabetes development acts in harmony with oxidative stress and chronic hyperglycemia to produce pathological alterations in different cells of peripheral nervous system including dorsal root ganglion neurons, neuronal axons and schwann cells. Due to contributory role of dyslipidemia in pathogenesis diabetic peripheral neuropathy, there is earlier incidence of peripheral neuropathy in type 2 was observed as compared to type 1 diabetes mellitus ${ }^{10}$.
In more than $50 \%$ of patients with diabetic neuropathy, irreversible nerve damage has been occurred prior to diagnosis. That leads to increase number of diabetes related hospital admission and limb amputations. Currently there is no effective treatment is present that completely cure diabetic neuropathy. So, research on different pathways involved inpathogenesis of diabetic neuropathy should be enhanced ${ }^{6}$.

Currently, strict glycemic control and symptomatic relieve are the only modalities to treat diabetic neuropathy and still, the prevalence of this diabetic complication is rising. Dyslipidemia is an emerging factor involved in pathogenesis of diabetic neuropathy. So, this study contributes to the better understanding and knowledge of the lipid patterns in the patients with diabetes mellitus, suffering with peripheral neuropathy and patients with diabetes mellitus without neuropathy complications.

\section{METHODOLOGY}

This comparative-cross sectional study was carried out between January 2020 to September 2020 in Multidisciplinary Lab-1 of Biochemistry \& Molecular Biology Department, Army Medical College in collaboration with Pak-Emirate Military Hospital, Rawalpindi. Research was conducted after formal approval of institutional ethics review committee.

Total eighty-four subjects were included in this study. Sample size was calculated using the World Health Organization (W.H.O) calculator based on reference prevalence $(6.9 \%)^{11}$, with $95 \%$ confidence interval and 5\% error. The study participants were recruited by non-probability, consecutive sampling technique and divided into three groups. Group I included 28 patients with diagnosed type 2 diabetes mellitus with peripheral neuropathy, group II includes 28 patients with diagnosed type 2 diabetes mellitus without neuropathy and group III comprised of 28 healthy control subjects. These subjects were enrolled after taking informed consent and clinical and demographic data obtained using a structured questionnaire. Patients with and without diabetic 
peripheral neuropathywere diagnosed by the medical specialists in PEMH Rawalpindi.

Patients with diabetic neuropathy and without diabetic neuropathy of both genders between the age group 30-70 years were included in the study and patients with non-diabetic neuropathy, pedal edema, lumbosacral pathology and limb amputations were excluded. Serum lipid profile included total cholesterol (TC), serum triglyceride
(36.9\%) females with mean age $50.75 \pm 10.37$ years of participants. Group I included $18(64.3 \%)$ males and 8 (35.7\%) females, group II included 20 males $(71.4 \%)$ and $8(28.6 \%)$ females, while group III included $15(53.6 \%)$ males and 13 (46.4\%) females. The mean values of TC, TAG, HDL-C and LDL-C with ANOVAare given in table-I. Group comparison by ANOVA followed by post-Hoc Tukey test is given in table-II.

Table-I: Mean values of lipid profile of all groups.

\begin{tabular}{l|c|c|c|c}
\hline Parameters & $\begin{array}{c}\text { Group I (Diabetic } \\
\text { with neuropathy) } \\
\text { Mean } \pm \text { SD }\end{array}$ & $\begin{array}{c}\text { Group II (Diabetics } \\
\text { without neuropathy) } \\
\text { Mean } \pm \text { SD }\end{array}$ & $\begin{array}{c}\text { Group III } \\
\text { (Healthy-controls) } \\
\text { Mean } \pm \text { SD }\end{array}$ & $\begin{array}{c}p \text {-value } \\
\text { Total cholesterol (TC) mmol/L }\end{array}$ \\
$4.87 \pm 1.6$ & $2.75 \pm 2.29$ & $2.45 \pm 0.94$ & $4.23 \pm 0.72$ & 0.102 \\
\hline Triglycerides (TAG) mmol/L & $1.02 \pm 0.22$ & $0.99 \pm 0.21$ & $1.31 \pm 0.52$ & $0.001^{*}$ \\
\hline $\begin{array}{l}\text { High-density lipoprotein } \\
\text { cholesterol (HDL-C) mmol/L }\end{array}$ & $3.07 \pm 1.11$ & $3.09 \pm 0.64$ & $2.81 \pm 0.71$ & $<0.01^{*}$ \\
\hline $\begin{array}{l}\text { Low-density lipoprotein } \\
\text { cholesterol (LDL-C) mmol/L }\end{array}$ & 0.361 \\
\hline
\end{tabular}

\begin{tabular}{|c|c|c|c|}
\hline \multirow{2}{*}{ Parameters } & \multicolumn{2}{|c|}{ Group I v/s } & \multirow{2}{*}{$\frac{\text { Group II v/s }}{\text { Group III }}$} \\
\hline & Group II & Group III & \\
\hline Triglycerides (TAG) & 0.714 & $0.001^{*}$ & 0.13 \\
\hline High-density lipoprotein cholesterol (HDL-C) & 0.872 & $0.003^{*}$ & $0.001^{*}$ \\
\hline Total cholesterol (TC) & 0.459 & 0.083 & 0.598 \\
\hline Low-density lipoprotein cholesterol (LDL-C) & 0.995 & 0.461 & 0.405 \\
\hline
\end{tabular}

(TAG), high-density lipoprotein cholesterol (HDL-C) and low-density lipoprotein cholesterol (LDL-C) was measured. Total $5 \mathrm{ml}$ of fasting (812 hours fasting) venous sample wasdrawn from eachparticipant. Lipid profile parameters were measured by Siemens Advia automated chemistry analyzer.

SPSS-22 (statistical package for social sciences-22) was used for data analysis. Categorical variables were expressed as percentage while quantitative variables were assessed as mean \pm standard deviation $( \pm \mathrm{SD})$. One-way ANOVA test was used followed by post-Hoc Tukey test and $p$ value $\leq 0.05$ was considered significant.

\section{RESULTS}

Total eighty-four subjects were enrolled in this study between the age-group of 30-70 years of both genders and divided into three groups. Among them there were $53(63.1 \%)$ males and 31
The mean value of triglycerides was significant $(<0.05)$ between group I and III and insignificant (0.714) between group I and II as well as (0.13) in group II and III. Triglycerides levels were higher in patients with diabetic neuropathy as compared to other groups. While the mean value of HDL-C was significant among group I and III as well as group II and III. Mean value of HDL-C was lowest in group II as compared to others. Mean value of total cholesterolwas high in group I as compared to group II and III, but that was not statistically significant. Similarly, mean value of LDL-C was higher in group II as compared to group I and III and it was non-significant. All parameters of lipid profile were statistically insignificant between group I and II.

\section{DISCUSSION}

Diabetic neuropathycauses malfunctioning of peripheral nerves which can progress to foot 
ulceration, ultimately leading to limb amputation. The main focus of this study was to evaluate lipid profile in patients with diabetic neuropathy and without neuropathy. The key findings obtained in this study were the presence of significant difference in triglyceride and HDL-C levels among patients with diabetic neuropathy, without neuropathy and healthy controls. However, no significant statistical difference was found among diabetics with neuropathy and without neuropathy. Considering laboratory results, there was significant variation in HDL-C levels between diabetics without neuropathy and healthy controls. However, no statistically significant difference was found regarding triglyceride levels between these groups.

In concordance to our study, similar results were reported by Mohapatra \& Damodar as they conducted the studyfor the evaluation of lipid profile and glycemic control among different stages of diabetic neuropathyand controls. They reported the significant reduction in HDL-C levels with increasing severity of diabetic neuropathy and increased triglyceride levels and increased LDL/HDL ratio were found in patients with diabetic neuropathyas compared to control'12. Another study compare lipid profile parameters between patients with diabetic neuropathy and diabetics without neuropathy and reported the findings consisted to our study that non-significant reduction in HDL-C and elevation in triglyceride levels among these groups ${ }^{13}$. On the other hand, a study conducted by Pai et al, on Taiwanese population, in which association of lipid profile was analyzed in patients with diabetic peripheral neuropathy with or without neuropathic pain. This study showed significant association of high level of triglycerides in patients with diabetic neuropathy without pain as compared to diabetics without neuropathy, while lower levels of HDL$\mathrm{C}$ were independently correlated patients with diabetic peripheral neuropathywith pain ${ }^{14}$.

Deranged lipid profile is also associated with other microvascular complications. A study conducted on Pakistani population by Amin et al, for comparison of lipid profile in patients with diabetes mellitus with retinopathy and without retinopathy. They found consistent result to our study that significant increase in triglyceride levels and reduction in HDL-C levels in patients withdiabetic retinopathy as compared to controls. There was also strong positive association of serum LDL-C, total cholesterol and triglyceride with severity of diabetic retinopathy ${ }^{15}$.

In this study, the mean value of total cholesterol was higher in patients with diabetic neuropathy as compared to other groups but was statistically not-significant. On the other hand, mean value of LDL-C was high in patients with diabetes mellitus without neuropathyas compared to other groups but it was also statistically non-significant. Lin et al, reported similar results that the high levels of total cholesterolin patients with diabetic neuropathy as compared to without neuropathy. But these findings were statistically significant in contrast to our study. A significant positive correlation between lipid profiles and HBA1c level was also observed in their study ${ }^{16}$.

In contrast to these results, Jende et al, reported in their study that serum cholesterol and LDL-C levels were low and correlated with peripheral nerve swelling in patients with diabetic peripheral neuropathy. So, patients with diabetes mellitus on lipid lowering therapy and with very low cholesterol levels should be vigilant regarding onset or further-worsening of neuropathic symptoms ${ }^{17}$. Contradictory to our study, another study also found statistically significant decreased levels of total cholesterol in patients with diabetic neuropathy as compared to without diabetic neuropathyand statically significant decrease levels of triglyceride in patients with DPN as compared to without DPN ${ }^{18}$. One study reported the significant reduction in the levels of LDL-C and total cholesterol and these were correlated with increasing severity of DPN stages ${ }^{12}$. Herder et al, reported significant increase levels of total cholesterol (0.002) and LDL-C (0.005) in patients without DSPN as compared to patients with DSPN19. A systemic review and meta-analysis carried by Naqvi et al, to evaluate the predicting value of LDL-C levels in patients withand with- 
out diabetic peripheral neuropathy. A significant correlation of reduced LDL-C with poor prognosis of DPN and diabetic foot was reported by them20. Aguiar et al, found significant association among total cholesterol and triglyceride levels and DPN. So, patients with diabetes mellitus having raised level of total cholesterol and triglyceride should be screened for neuropathy ${ }^{21}$. Increased levels of LDL-C were observationally and genetically correlated with increase risks of peripheral arterial disease and chronic kidney disease in a study conducted by Emanuelsson et al, and they also reported increased levels of LDL-C were not correlated with retinopathy and neuropathy in patients with diabetes mellitus ${ }^{22}$.

\section{ACKNOWLEDGMENT}

We thanks to Dr. Asifa Majeed, Assoc Prof, Department of Biochemistry and Molecular Biology who provided insight and expertise that greatly assisted the research and shared her pearls of wisdom with us during the course of this research.

\section{CONCLUSION}

Serum triglyceride levels were significantly increased and high-density lipoprotein cholesterol levels were significantly decreased in patients with diabetic neuropathyas compared to healthy controls. There was no significant difference was observed regarding all lipid profile parameters between patients with diabetic neuropathy and without diabetic neuropathy.

\section{CONFLICT OF INTEREST}

This study has no conflict of interest to be declared by any author.

\section{REFERENCES}

1. Saeedi $P$, Petersohn I, Salpea $P$, Malanda B, Karuranga S, Unwin $\mathrm{N}$, et al. Global and regional diabetes prevalence estimates for 2019 and projections for 2030 and 2045: Results from the International Diabetes Federation Diabetes Atlas. Diabetes Res Clin Pract 2019; 157(1): 107843.

2. Valencia WM, Florez H. How to prevent the microvascular complications of type 2 diabetes beyond glucose control. BMJ 2017; 356(2): I6505.

3. Dewanjee S, Das S, Das AK, Bhattacharjee N, Dihingia A, Dua TK. Molecular mechanism of diabetic neuropathy and its pharmacotherapeutic targets.Eur J Pharmacol 2018; 833(2): 472-23.
4. Pop-Busui R, Boulton AJ, Feldman EL, Bril V, Freeman R, Malik RA, et al. Diabetic neuropathy: a position statement by the american diabetes association. Diabetes Care 2017; 40(1): 136-54.

5. Liu X, Xu Y. The risk factors for diabetic peri-pheral neuropathy: A meta-analysis. PLoS One 2019; 14(2): e0212574.

6. Feldman EL, Nave KA, Jensen TS, Bennett DL. New horizons in diabetic neuropathy: mechanisms, bioenergetics, and pain. Neuron 2017; 93(6): 1296-13.

7. Teodoro JS, Nunes S, Rolo AP, Reis F, Palmeira CM. Therapeutic options targeting oxidative stress, mitochondrial dysfunction and inflammation to hinder the progression of vascular complications of diabetes.Front Physiol 2019; 9(1): 1857-60.

8. Schofield JD, Liu Y, Rao-Balakrishna P, Malik RA, Soran H. Diabetes dyslipidemia. Diabetes Ther 2016; 7(2): 203-19.

9. Eid S, Sas KM, Abcouwer SF, Feldman EL, Gardner TW. New insights into the mechanisms of diabetic complications: role of lipids and lipid metabolism. Diabetol 2019; 62(9): 1539-49.

10. Callaghan BC, Gallagher G, Fridman V. Diabetic neuropathy: what does the future hold. Diabetol 2020; 63(5): 891-97.

11. Bukhsh A, Khan TM, Nawaz MS, Ahmed HS, Chan KG. Association of diabetes knowledge with glycemic control and self-care practices among Pakistani people with type 2 diabetes mellitus. Diabetes Metab Syndr Obes 2019; 12(1): 1409-12.

12. Moha Patra D, Da Mo-Dar KS. Glycaemia status, lipid profile and renal parameters in progressive diabetic neuropathy. J Clin Diagn Res 2016; 10(9): CC14.

13. Buraczynska M, Buraczynska K, Dragan M, Ksiazek A. Pro198 Leu polymorphism in the glutathione peroxidase 1 gene contributes to diabetic peripheral neuropathy in type 2 diabetes patients. Neuromol Med 2017; 19(1): 147-53.

14. Pai YW, Lin CH, Lee IT, Chang MH. Prevalence and biochemical risk factors of diabetic peripheral neuropathy with or without neuropathic pain in Taiwanese adults with type 2 diabetes mellitus. Diabetes Metab Syndr 2018; 12(2): 111-16.

15. Amin ZA, Islam QU, Mehboob MA. Comparison of serum lipid profile in Type-2 Diabetics with and without retinopathy in Pakistani population. Pak J Med Sci 2016; 32(6): 1349-52.

16. Lin X, Xu L, Zhao D, Luo Z, Pan S. Correlation between serum uric acid and diabetic peripheral neuropathy in T2DM patients. J Neurol Sci 2018; 385(2): 78-82.

17. Jende JM, Groener JB, Rother C, Kender Z. Association of serum cholesterol levels with peripheral nerve damage in patients with type 2 diabetes. JAMA Netw Open 2019; 2(5): e194798.

18. Yang J, Yan PJ, Wan Q, Li H. Association between Hemoglobin Levels and Diabetic Peripheral Neuropathy in Patients with Type 2 Diabetes: A Cross-Sectional Study Using Electronic Health Records. J Diabetes Res 2017; 2017(1): 2835981.

19. Herder C, Kannenberg JM, Huth C, Kirberg MC, Rathmann W. Myeloperoxidase, superoxide dismutase 3, car-diometabolic risk factors, and distal sensorimotor polyneuropathy: The KORA F4/FF4 study. Diabetes Metab Res Rev 2018; 34(5): e3000.

20. Naqvi SSZH, Imani S, Hosseinifard H, Wen QL, Shahzad MN, Ijaz I, et al. Associations of serum low-density lipoprotein and systolic blood pressure levels with type 2 diabetic patients with and without peripheral neuropathy: systemic review, metaanalysis and meta-regression analysis of observational studies. BMC Endocr Disord 2019; 19(1): 125-28.

21. Aguiar P, Coletta MD, de Souza J. The association of dyslipidemia and peripheral diabetic neuropathy: The influence of urea. Diabetol Metab Syndr 2016; 1(2): 2-5.

22. Emanuelsson F, Nordestgaard BG. Impact of LDL cholesterol on microvascular versus macro-vascular disease: a Mendelian randomization study. J Am Coll Cardiol 2019; 74(11): 1465-76. 